\title{
Energy Efficient Entropy Based Clustering in Wireless Sensor Networks
}

\author{
Rajeev Kumar, Naveen Chauhan and Narottam Chand \\ Department of Computer Science \& Engineering \\ National Institute of Technology, Hamirpur (India) \\ \{rajeev,naveen,nar\}@nith.ac.in
}

\begin{abstract}
Wireless sensor networks usually consist of a large group of cooperating wireless sensor nodes deployed in area of interest. These sensor nodes are having very limited resources in terms of the energy, the bandwidth, the capability of computing, etc. Most crucial of these resources is battery life, as in most applications like battle field or volcanic area monitoring, it is often impossible to replace or recharge the power source. This paper presents an energy efficient clustering algorithm based on entropy. Clustering has always been a basic method to organize large number of sensor nodes into suitable groups. We divide the network into preliminary grids depending on the spatial correlation of sensor node. These grids are then modified by applying a dynamic scheme, where the change of sensor data determine which cluster a sensor node to join. On the basis of results, it is safe to conclude that clustering in wireless sensor networks using entropy improves the life time of nodes in the networks thereby prolonging the network's life time.
\end{abstract}

Keywords: Clustering; Average Entropy; WSNs; Clustering algorithms; Scalability.

\section{Introduction}

Wireless sensor networks (WSNs) consists of a large number of low cost scalar nodes, which are small in size and deployed in hostile environment. In WSNs, sensor nodes deal with collecting, processing and communicating sensed data over wireless medium. These nodes are battery driven devices [1], and it is hard or rather impossible to replace the batteries in inaccessible or hostile environment. WSNs are resource constrained, so it is expected that these nodes perform their tasks for the maximum time period. Sensor nodes are typically disposable and expected to last until their energy drains. Therefore, energy is a very scarce resource for such sensor nodes and has to be managed intelligently in order to extend the life of the sensor nodes. WSNs are employed to monitor certain data in an environment. Sensor nodes will collect data in the region, process it and collected data is transmitted to the sink node, which is used by the end user. In contrast to traditional networking, a WSNs has its own design and resource limitations. Resource limitations include a limited amount of energy, short communication range, low bandwidth, and limited processing and storage in each sensor node. Design constraints are application dependent and are based on the monitored environment. The environment plays a key role in determining the size of the network, the distribution scheme of sensor nodes, and the network topology. The size of the network varies with the monitored environment. For enclosed environments, fewer nodes are required to form a network in a limited space whereas open-air environments may require more nodes to cover a larger area. An ad hoc deployment is preferred over preplanned deployment when the environment is inaccessible by humans or when the network is composed of hundreds to thousands of nodes. Geographical impediments in the environment can also limit communication between nodes, which in turn affects the network connectivity. 
There are several applications of WSNs such as military target tracking and surveillance, natural disaster relief, biomedical health monitoring, weather monitoring and early warning, resource management and hazardous environment exploration and seismic sensing [1]. Most of WSNs applications are data centric. In these applications sensor nodes transmit the sensed data to the sink through single/multi hop transmission. The sink node is located close or inside/outside the sensing field. Sink collects data and performs some specific processing accordance with the application requirement and then transmits to the end user. Large numbers of nodes are deployed in sensing field. The large volume of data is transmitted and received by sink that leads to higher energy consumption.

Rest of the paper is summarized as follows. Section 2 describes the work related to clustering of sensor nodes, where concept of entropy is used. Entropy theory is explained in section 3. Proposed protocol has been explained in Section 4. Section 5 defines various simulation parameters, performance metrics and explains simulation results. Section 6 explains the conclusion of the work.

\section{Related Work}

Clustering algorithms in the literature vary in their objectives. Entropy has been widely used in clustering in wireless sensor networks. Wang et el. [2] present EECRU, an energy efficient clustering method that uses random update. The authors assume that network is initially divided into clusters based upon temporal and spatially correlation of data. Authors also present cluster head rotation scheme using dynamic updates. An entropy based clustering hierarchy for wireless sensor networks (E-BACH) [3] concentrates on network optimization using data quality which is based upon the data entropy of individual sensor node. Nga et el. [4] proposed a clustering method for sensor node using correlation among the regions. To define the correlation region, the joint entropy of multiple sensed data is calculated. After calculation of joint entropy, clustering is performed in those correlated regions. Xiao et el. [5] consider the problem of appropriate cluster head selection and proposed an energy entropy based cluster head selection algorithm for wireless senor networks (EE-CSAW). The authors construct a new metric called node stability to select a stable cluster head to reduce the number of re-clustering process. Sinha and Lobiyal [6] proposed a dynamic clustering and aggregation method for wireless sensor networks. In proposed method, the data is aggregated at sensor node as well as on the cluster head. The authors reduced the number of transmissions and processing cost using entropy theory while maintaining the relevance of aggregated data.

\section{Entropy Theory}

The entropy is a measure of the average uncertainty in the random variable [7]. The entropy of a random variable $\mathrm{X}$ with a probability mass function $\mathrm{p}(\mathrm{x})$ is defined by Equation 1.

$$
H(x)=-\sum p(x) \log _{2} p(x)
$$

Conditional entropy $\mathrm{H}(\mathrm{X} \mid \mathrm{Y})$, is the entropy of a random variable $\mathrm{Y}$ conditional on the knowledge of another random variable $\mathrm{X}$. The reduction in uncertainty due to another random variable is called the mutual information. The mutual information $\mathrm{I}(\mathrm{X} ; \mathrm{Y})$ is a measure of the dependence between the two random variables. It is symmetric in $\mathrm{X}$ and $\mathrm{Y}$ and always non-negative and is equal to zero if and only if $\mathrm{X}$ and $\mathrm{Y}$ are independent. For two random variables $\mathrm{X}$ and $\mathrm{Y}$ the mutual information where joint distribution is defined by $\mathrm{p}(\mathrm{x}, \mathrm{y})$ is given by Equation 2 .

$$
I(X ; Y)=H(X)-H(X \mid Y)=\sum_{x, y} p(x, y) \log _{2} \frac{p(x, y)}{p(x) p(y)}
$$


The joint entropy $\mathrm{H}(\mathrm{X}, \mathrm{Y})$ of a pair of discrete random variables $(\mathrm{X}, \mathrm{Y})$ with a joint distribution $\mathrm{p}(\mathrm{x}, \mathrm{y})$ is defined by Equation 3.

$$
H(X, Y)=-\sum_{x \in X} \sum_{y \in Y} p(x, y) \log _{2} p(x, y)
$$

Conditional entropy of a random variable given another as the expected value of the entropies of the conditional distributions, is obtained as averaged over the conditioning random variable by Equation 4 .

$$
H(X \mid Y)=-\sum_{x \in X} \sum_{y \in Y} p(x, y) \log _{2} p(x \mid y)
$$

The naturalness of the definition of joint entropy and conditional entropy is exhibited by the fact that the entropy of a pair of random variables is the entropy of one plus the conditional entropy of the other. In Wang et al. [2] introduce the concept of average entropy using Slepian-Wolf theorem. For any node $s_{i}, s_{j} \in S$, average entropy is defined as Equation 5.

$$
\mathrm{AH}_{\mathrm{s}_{i}} \approx \sum_{j} \frac{H\left(s_{j} \mid s_{i}\right)}{|S|}
$$

Average entropy gives the average data rate from every node in $S$ to their center node $s_{i}$. Further Wang et al. [2] applied linear correlation coefficient $\rho_{s_{i} s_{j}}$ to estimate the value of average entropy which is expressed by Equation 6 .

$$
\mathrm{AH}_{\mathrm{s}_{i}} \approx \sum_{j} \frac{\left(1-\frac{\rho_{s_{i} s_{j}}}{2}\right)\left(H\left(s_{i}\right)+H\left(s_{j}\right)\right)-H\left(s_{i}\right)}{\mid \text { Number of sensor nodes in the neighbhor of } s_{i} \mid}
$$

\section{Proposed Work}

In this section, we described our proposed energy efficient entropy based clustering (E3C) in detail.

\subsection{Grid Formation}

Here, we propose an entropy based clustering protocol. Proposed protocol is based on spatial and temporal correlation among the sensor nodes. Initially whole sensor field is divided in to virtual grids. Each grid is of size $1 \times 1$ as shown in Figure 1.

Every grid has Grid Id $G_{x, y}$. For a node $s_{i}$ with coordinates $(x, y)$, the grid id is computed as follows:

$\mathrm{G}_{\mathrm{x} \mathrm{y}}=\left(\left\lfloor\frac{x}{l}\right\rfloor,\left\lfloor\frac{y}{l}\right\rfloor\right)$, where $\lfloor\mathrm{k}\rfloor$ is the largest integer less than equal to $\mathrm{k}$. After splitting the sensor field into equal sized grids, one cluster head will be selected in each grid.

The initial selection of cluster head $(\mathrm{CH})$ within a grid is based upon the choice of a node that is located nearest to the centroid of the grid. The centroid $\left(\mathrm{x}_{\mathrm{c}}, \mathrm{y}_{\mathrm{c}}\right)$ of a node $\mathrm{s}_{\mathrm{c}}$ inside a grid with Grid ID $\mathrm{G}_{\mathrm{x}, \mathrm{y}}$ is given as $X_{c}=\left(\frac{G_{x}+G_{x-1}}{2}\right) * l, Y_{c}=\left(\frac{G_{y}+G_{y-1}}{2}\right) * l$. Each node $\mathrm{s}_{\mathrm{i}}$ with coordinates $(\mathrm{x}, \mathrm{y})$ calculates its distance $\mathrm{d}_{\mathrm{i}}$ from the centroid $\left(\mathrm{X}_{\mathrm{c}}, \mathrm{Y}_{\mathrm{c}}\right)$ as $d_{i}=\sqrt{\left(X_{c}-x\right)^{2}+\left(Y_{c}-y\right)^{2}}$. We use the concept of back off timer to select a $\mathrm{CH}$. For a node $\mathrm{s}_{\mathrm{i}}$, the back off timer $\mathrm{t}_{\mathrm{i}}$ is set as $t_{i}=d_{i} * B_{t}$ where $\mathrm{B}_{\mathrm{t}}$ is a random number 
whose value is in the range $(0.9,1)$. Each node $s_{\mathrm{i}}$ broadcasts its $t_{\mathrm{i}}$ value and a node with least back off time $t_{i}$ is selected as $\mathrm{CH}$. Further the proposed entropy based clustering technique exploits temporal correlation among the sensor nodes.

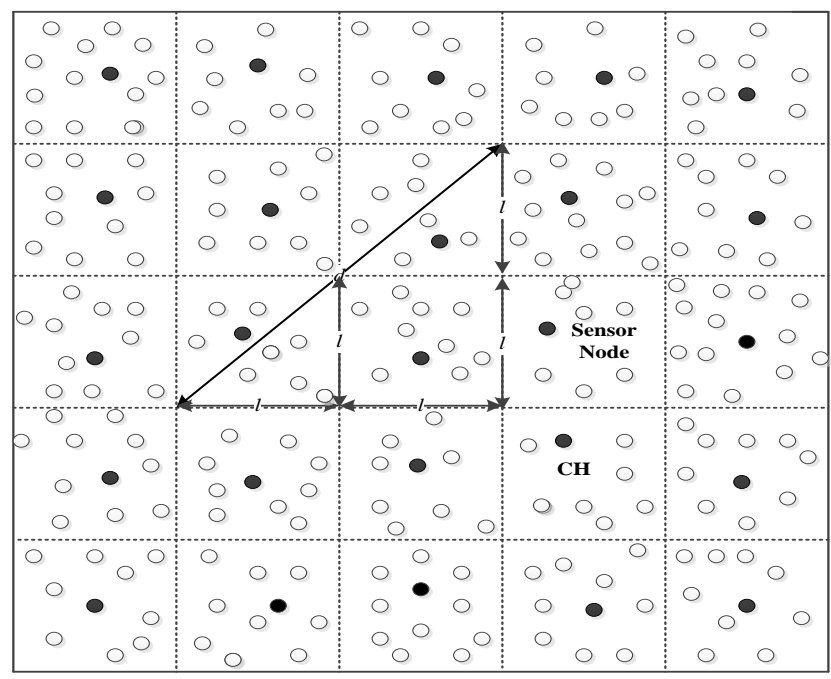

Figure 1. Formation of Virtual Grids in a WSN

\subsection{Identifying Boundary Nodes}

During grid formation, sensor field is divided in virtual grids and node near to centroid is selected as initial cluster head. Node near to centroid will be more spatially correlated to cluster head as compare to nodes near to boundary of grid. Now nodes near boundary can be correlated to adjacent grids. Thus, there will be some fragile region at boundary of each grid where grid nodes may exhibit correlation with adjacent grid as shown in Figure 2.

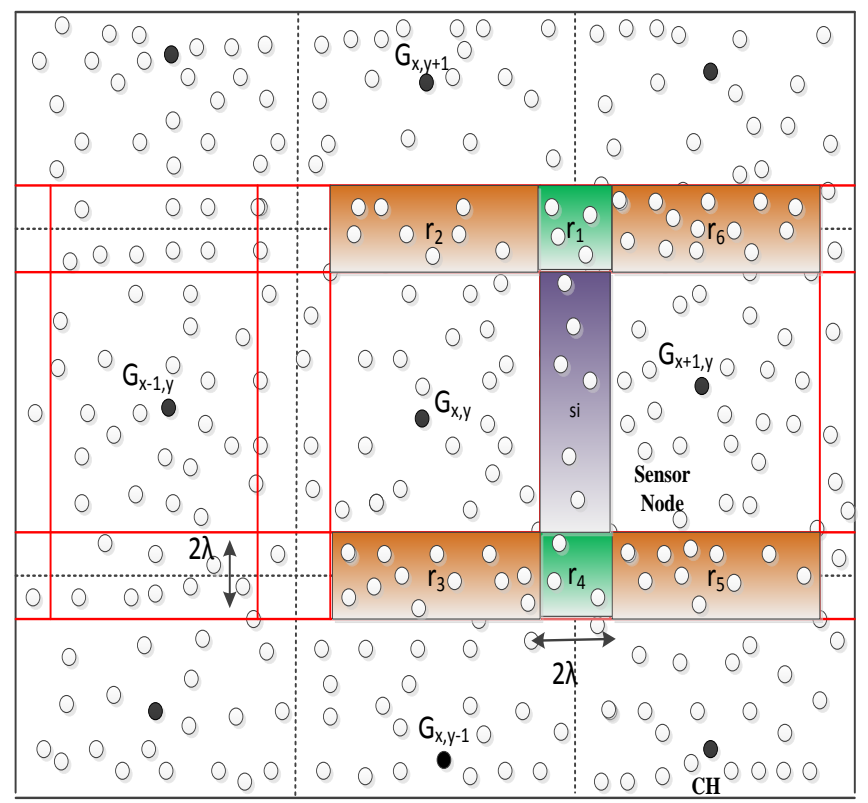

Figure 2. Sensor Nodes at Boundary 
Let us consider a node $s_{i}$ at the boundary of grid $G_{x, y}$. Sensor node $s_{i}$ can be part of the any of adjacent grids. To decide about $\mathrm{s}_{\mathrm{i}}$, we divide the adjacent grids into different regions with respect to sensor node $\mathrm{s}_{\mathrm{i}}$. The coordinates of neighboring regions are as follows as shown in Table 1.

Table 1. Coordinates of Neighboring Regions

\begin{tabular}{|l|l|}
\hline Neighboring region & Coordinates with reference to centroid of $\mathrm{G}_{\mathrm{x}, \mathrm{y}}$ as $(0,0)$ \\
\hline $\mathrm{nr}_{1}$ & $\left(\frac{l}{2}-\lambda, \frac{l}{2}-\lambda\right),\left(\frac{l}{2}+\lambda, \frac{l}{2}+\lambda\right)$, \\
\hline $\mathrm{nr}_{2}$ & $\left(-\frac{l}{2}+\lambda, \frac{l}{2}-\lambda\right),\left(\frac{l}{2}-\lambda, \frac{l}{2}+\lambda\right)$ \\
\hline $\mathrm{nr}_{3}$ & $\left(-\frac{l}{2}+\lambda,-\frac{l}{2}-\lambda\right),\left(\frac{l}{2}-\lambda,-\frac{l}{2}+\lambda\right)$ \\
\hline $\mathrm{nr}_{4}$ & $\left(\frac{l}{2}-\lambda,-\frac{l}{2}-\lambda\right),\left(\frac{l}{2}+\lambda,-\frac{l}{2}+\lambda\right)$ \\
\hline $\mathrm{nr}_{5}$ & $\left(\frac{l}{2}+\lambda,-\frac{l}{2}-\lambda\right),\left(2 l-\lambda,-\frac{l}{2}+\lambda\right)$ \\
\hline $\mathrm{nr}_{6}$ & $\left(\frac{l}{2}+\lambda, \frac{l}{2}-\lambda\right),\left(2 l-\lambda, \frac{l}{2}+\lambda\right)$ \\
\hline
\end{tabular}

Table 2. Symbols Used

\begin{tabular}{|l|l|}
\hline Symbol & Meaning \\
\hline $\mathrm{H}\left(\mathrm{s}_{\mathrm{i}}\right)$ & Entropy of sensor node $\mathrm{s}_{\mathrm{i}}$ \\
\hline $\mathrm{AH} \mathrm{H}_{\mathrm{s}_{i}}^{k}$ & Average entropy of $\mathrm{s}_{\mathrm{i}}$ with region $\mathrm{k}$ \\
\hline$\lambda$ & User Specified Boundary factor \\
\hline$\rho_{s_{i} s_{j}}$ & Pearson's linear correlation coefficient among sensor node $\mathrm{s}_{\mathrm{i}}$ and $\mathrm{s}_{\mathrm{j}}$ \\
\hline $\mathrm{V}_{\text {avg }}^{G_{j}}$ & Average value of members of grid $\mathrm{G}_{\mathrm{j}}$ \\
\hline
\end{tabular}

\footnotetext{
Algorithm 1: Cluster Formation

$1 \quad$ Each node sends its data value to the grid head

2 Each grid head computes average entropy

$$
\mathrm{AH}_{\mathrm{G}_{j}} \approx \sum_{j} \frac{\left(1-\frac{\rho_{s_{i} s_{j}}}{2}\right)\left(H\left(s_{i}\right)+H\left(s_{j}\right)\right)-H\left(s_{i}\right)}{\mid \text { Number of sensor nodes in Grid } \mathrm{G}_{j} \mid}
$$

3 Grid head computes average value $\mathrm{V}_{\text {avg }}^{G_{j}}$

4 Grid head broadcasts $\mathrm{V}_{a v g}^{G_{j}}$ and $\mathrm{AH}_{\mathrm{G}_{j}}$ to its grid members

$5 \quad$ For each node $s_{x}$ that belongs to all region $r_{i}$ in adjacent grids with boundary factor $\lambda$

6 Compute $\mathrm{H}\left(\mathrm{V}_{\text {avg }}^{G_{j}} \mid s_{x}\right)-\mathrm{AH}_{\mathrm{G}_{j}}$ for node $\mathrm{s}_{\mathrm{x}}$ for all neighboring grids

$7 \quad$ Node $\mathrm{s}_{\mathrm{x}}$ joins grid $G_{k}$ such that $\mathrm{H}\left(\mathrm{V}_{a v g}^{G_{k}} \mid s_{x}\right)-\mathrm{AH}_{\mathrm{G}_{k}}$ is minimum.

8 Associate node $\mathrm{s}_{\mathrm{i}}$ with grid $\mathrm{G}_{\mathrm{k}}$

9 Expanded $\mathrm{G}_{\mathrm{k}}$ is now new cluster $\mathrm{C}_{\mathrm{k}}$

10 Broadcast $\mathrm{CH}$ information among its members
} 


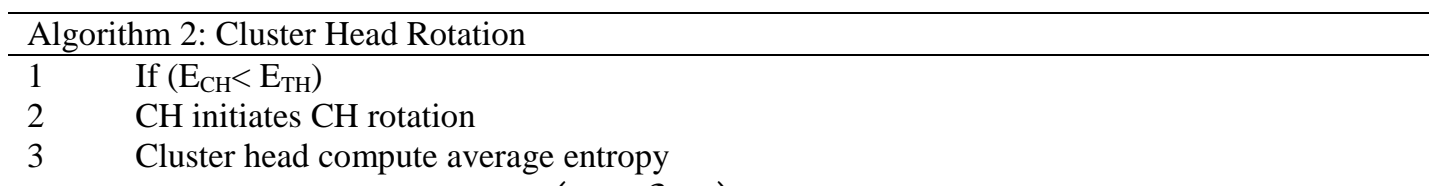

Cluster head compute average value $\mathrm{V}_{\text {avg }}^{C_{j}}$

$$
\mathrm{AH}_{C_{j}} \approx \sum_{j} \frac{\left(1-\frac{\rho_{s_{i} s_{j}}}{2}\right)\left(H\left(s_{i}\right)+H\left(s_{j}\right)\right)-H\left(s_{i}\right)}{\mid \text { Number of sensor nodes in cluster } C_{j} \mid}
$$

5

Cluster head broadcast $\mathrm{V}_{a v g}^{G_{j}}$ and $\mathrm{AH}_{\mathrm{G}_{j}}$ to its members

$6 \quad$ Each node $\mathrm{s}_{\mathrm{y}} \in \mathrm{C}_{\mathrm{k}}$ having $\left(\mathrm{E}>\mathrm{E}_{\mathrm{TH}}\right)$

7 Calculate EED value

$$
\operatorname{EED}\left(s_{y}\right)=\frac{\operatorname{Energy}\left(s_{y}\right)}{\mathrm{AH}_{C_{k}} \times \operatorname{Distance}\left(C_{k}-s_{y}\right)}
$$

8 Send EED to cluster head $C_{k}$

$9 \mathrm{CH}$ will select a sensor node $\mathrm{s}_{\mathrm{j}}$ with highest EED value as a new cluster head

10 Broadcast $\mathrm{s}_{\mathrm{j}}$ as a new cluster head

\subsection{Cluster Formation in Energy Efficient Entropy Based Clustering (E3C)}

This section describes the cluster formation based upon entropy. Initially sensor field is divided into virtual grids, and each grid has its grid head as described in previous section. Nodes nearer to the grid head are spatially correlated to the grid head, and likely to be the part of the same cluster, whereas nodes at boundaries with boundary factor $\lambda$ may be part of any of the neighboring grid as they possess equivalent degree of correlation with these neighboring grid as shown in Figure 2. Initially, each sensor node sends its value to grid head. Grid head computes average and also computes average entropy. After computing, grid head broadcasts average and average entropy to all sensor nodes under its communication range. To calculate average entropy following equation is used.

$$
\mathrm{AH}_{\mathrm{G}_{j}} \approx \sum_{j} \frac{\left(1-\frac{\rho_{s_{i} s_{j}}}{2}\right)\left(H\left(s_{i}\right)+H\left(s_{j}\right)\right)-H\left(s_{i}\right)}{\mid \text { Number of sensor nodes in Grid } \mathrm{G}_{j} \mid}
$$

To decide the membership of the sensor node located at boundary, each node $s_{\mathrm{x}}$ that belongs to region $r_{i}$ in adjacent grids with boundary factor $\lambda$, calculates $H\left(V_{a v g}^{G_{k}} \mid s_{x}\right)-$ $\mathrm{AH}_{\mathrm{G}_{k}}$. Node $\mathrm{s}_{\mathrm{x}}$ joins a grid $\mathrm{G}_{\mathrm{k}}$ if $\mathrm{H}\left(\mathrm{V}_{\text {avg }}^{G_{k}} \mid s_{x}\right)-\mathrm{AH}_{\mathrm{G}_{k}}$ is minimum. After deciding membership of all the boundary nodes, all grids are updated to clusters, with existing gird head as their new cluster head. New cluster formed will be more spatially and temporally correlated. Keeping existing grid head as a cluster head in new clusters, will save energy. The information of the cluster head will be broadcasted among the cluster members. Algorithm 1 gives the steps of cluster formation.

\subsection{Cluster Head Rotation in E3C}

To maintain the energy balance in cluster, we propose cluster head rotation among cluster members based upon entropy. Cluster head rotation process is initiated by current cluster head if the current level of cluster head is less than threshold level. Cluster head computes average entropy using Equation 8. 


$$
\mathrm{AH}_{C_{j}} \approx \sum_{j} \frac{\left(1-\frac{\rho_{s_{i} s_{j}}}{2}\right)\left(H\left(s_{i}\right)+H\left(s_{j}\right)\right)-H\left(s_{i}\right)}{\mid \text { Number of sensor nodes in cluster } C_{j} \mid}
$$

After calculation of average entropy, cluster head broadcasts $\mathrm{AH}_{C_{j}}$ to its members. Each cluster member calculates EED value using Equation 9.

$$
\operatorname{EED}\left(s_{i}\right)=\frac{\operatorname{Energy}\left(s_{i}\right)}{\mathrm{AH}_{\mathrm{s}_{i}} \times \operatorname{Distance}\left(\mathrm{CH}-\mathrm{s}_{i}\right)}
$$

After computing EED, each senor node sends its EED value to current cluster head. Current cluster head will select a sensor node $s_{j}$ with highest EED value as a new cluster head. New cluster head rotation message will be broadcasted to all other members of the cluster. Algorithm 2 gives the steps involved cluster head rotation.

\section{Performance Evaluation}

In this section, we evaluate the performance of proposed algorithm Energy Efficient Entropy based Clustering (E3C) through simulating in MATLAB. We first define simulation parameters, and performance metrics used. We then see the effect of various factors like transmission range, number of nodes and network size on performance metrics to measure the effectiveness of proposed algorithm. Simulation results are compared with GBS, where grid type clustering is used based upon spatial and temporal correlation among the nodes [8].

\subsection{Simulation Parameters}

We consider a flat and square sized two dimensional sensor field of size $200 \mathrm{~m} \times 200 \mathrm{~m}$ in which sensor nodes are randomly deployed. All nodes are homogeneous. Various simulation parameters are listed in Table 3.

\begin{tabular}{|c|c|c|}
\hline Parameter & Default Value & Range \\
\hline Network size (side of square sensor field) & $200 \mathrm{~m}$ & $50 \mathrm{~m} \sim 300 \mathrm{~m}$ \\
\hline Number of nodes & 400 & $100 \sim 500$ \\
\hline Transmission range $(\mathrm{R})$ & $100 \mathrm{~m}$ & $40 \mathrm{~m} \sim 140 \mathrm{~m}$ \\
\hline Initial energy of node & 2 Joule & \\
\hline Sink location & $(0,0)$ & \\
\hline Data packet size & $64 \mathrm{~KB}$ & \\
\hline $\mathrm{E}_{\text {elec }}$ & $50 \mathrm{~nJ} / \mathrm{bit}$ & \\
\hline$\varepsilon_{\mathrm{fs}}$ & $10 \mathrm{pJ} / \mathrm{bit} / \mathrm{m}^{2}$ & \\
\hline$\varepsilon_{\mathrm{mp}}$ & $0.00134 \mathrm{pJ} / \mathrm{bit} / \mathrm{m}^{4}$ & \\
\hline Data rate & $512 \mathrm{Kbps}$ & \\
\hline$\lambda$ & $\begin{array}{l}\text { User Specified } \\
\text { Boundary factor }\end{array}$ & $1 \sim 2$ \\
\hline
\end{tabular}

Table 3. Simulation Parameters

\subsection{Performance Metrics}

Following performance metrics are used to evaluate the performance of proposed protocol: 


\section{Network lifetime}

It is the time from deployment of sensor network till it can maintain its desired functionality, such as maintain desired connectivity and coverage. Based on desired functionality network lifetime has different definitions. During our simulation, we have used the following parameters to measure network lifetime:

a) FND: number of rounds after which first node dies.

b) HND: number of rounds after which $50 \%$ nodes die.

\section{Total Energy Dissipation per Round}

It is the energy dissipated within sensor network for sensing the environment and communicating the sensed data to the sink in one data collection and dissemination round. Smaller the energy consumed per round, better is the network lifetime.

\subsubsection{Effect of Transmission Range on Network Lifetime}

To see the effect of transmission range on the network lifetime, the number of nodes and the network size are kept fixed at their default values. Simulation results have been compared with grid based scheme [8] so-called GBS, where grid type clustering is used based upon spatial correlation among the sensor nodes. From the Figures 3(a) and $3(\mathrm{~b})$, it is observed that when the transmission range of node is small, the WSN is divided into large number of smaller grids because the grid size is directly related to the transmission range of sensor node. Therefore, the average number of hops for the sensed data to reach the sink increases which results in more consumption of energy. As a result of which the number of rounds for the FND and HND are less.

With the increase in transmission range, the grid size increases which decreases average number of hops for the sensed data to reach the sink. Because of this the energy consumption decreases and the number of rounds for the first node to die and half the nodes to die increase. At this stage, an optimal transmission range is achieved.

From the simulation, it has been observed that the transmission range of node should be between $100 \mathrm{~m}$ to $140 \mathrm{~m}$ in order to optimize the network lifetime. Results of E3C are better than GBS because in GBS cluster heads are elected after every round so the frequency of head rotation is quite high. Moreover, in GBS all the nodes participate in cluster head re-election process irrespective of their current residual energy, whereas in E3C only current $\mathrm{CH}$ and nodes having energy greater than threshold level are responsible to elect the new cluster head as discussed in Section 4.6, thus E3C gives better results than GBS approach. 


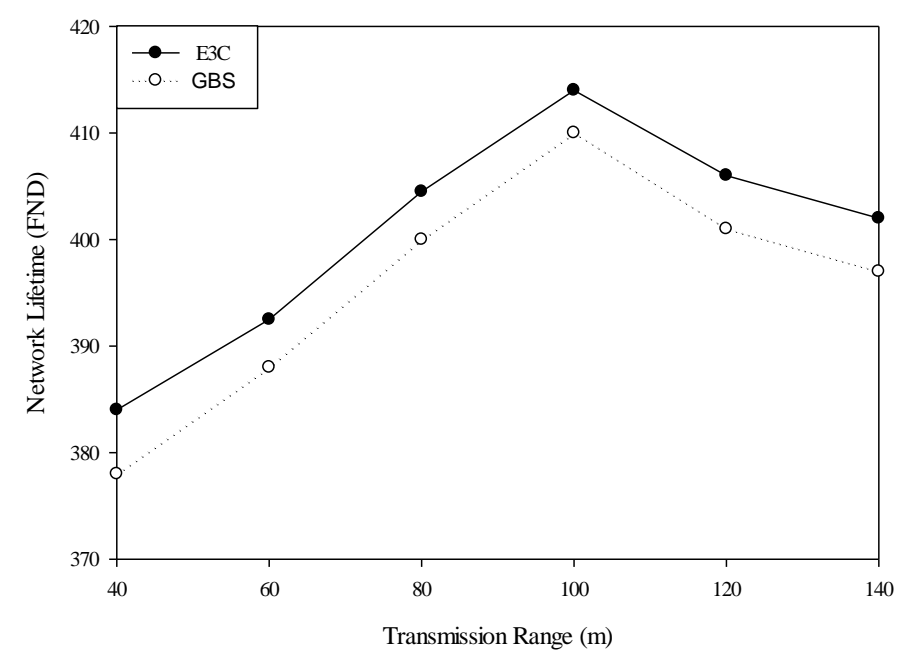

(a)

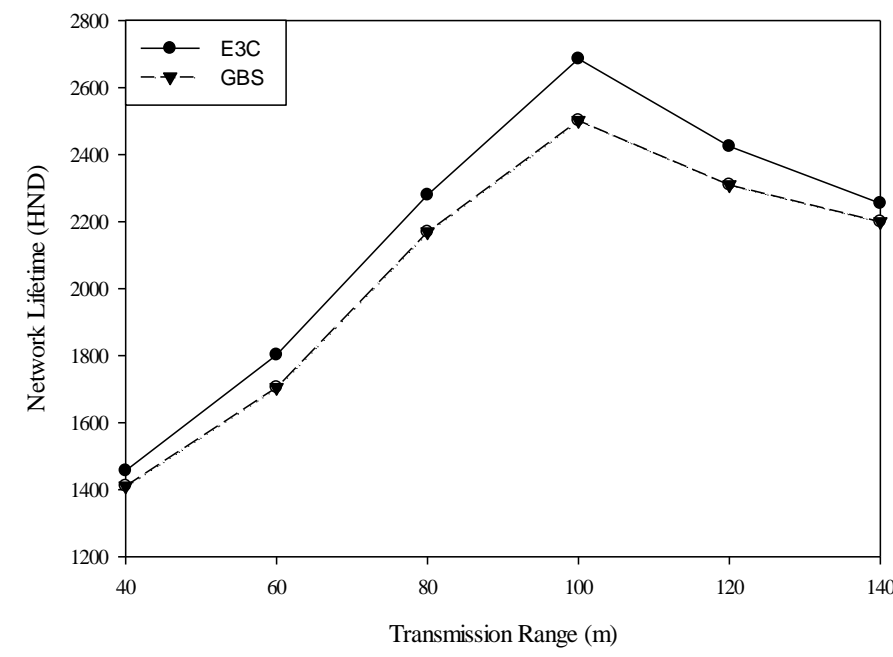

(b)

Figure 3. Effect of Transmission Range on (a) FND, and (b) HND

\subsubsection{Effect of Transmission Range on Network Latency}

Result of network latency as a function of transmission range of sensor nodes is shown in Figure 4. From the graph, it has been observed that when the transmission range of sensor node is small, the number of hops for the data transmission from sensor node to the cluster head and from cluster head to sink are more and therefore the time taken by the data to reach the sink is more. With the increase in transmission range, the number of hops decreases which results in decrease in the data transmission time and network latency. With the further increase in transmission range, sensor node will be able to reach the cluster head or to the sink in almost one hop distance and hence results in constant data transmission time which makes the network latency to appear almost same after a transmission range of around $100 \mathrm{~m}$. E3C results are better than GBS approach. 


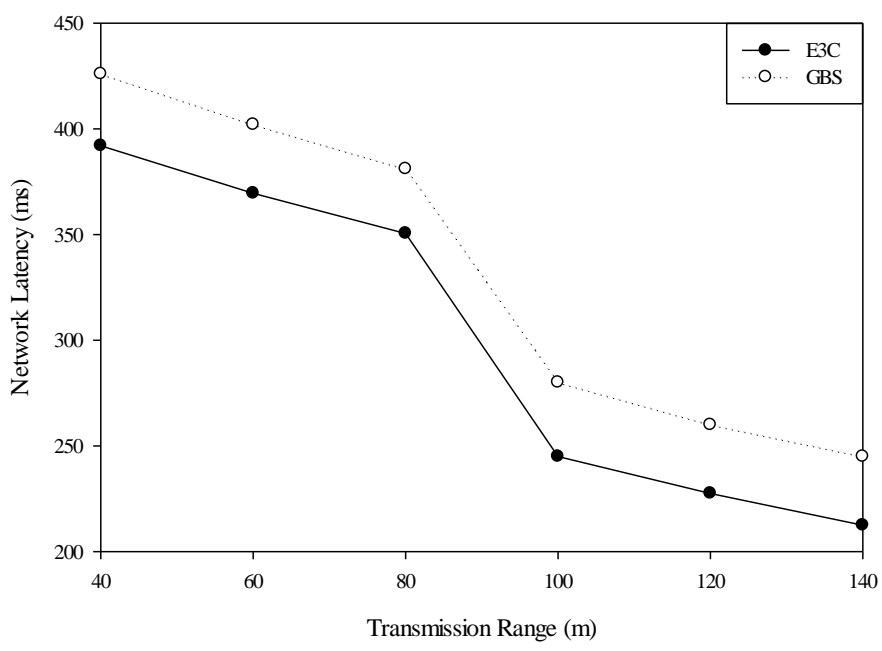

Figure 4. Effect of Transmission Range on Network Latency

\subsubsection{Effect of Transmission Range on Energy Consumption}

With the increase in transmission range, some data communications are subject to $\mathrm{d}^{4}$ attenuation, but with the increase in transmission range the grid size increases and the average number of hops for the transfer of data to sink decreases and also more redundant nodes can be put to sleep mode in larger clusters. Therefore, energy consumption decreases with increase in transmission range. At this stage, an optimal transmission range is achieved. If the range continues to increase further, the energy consumption associated with transmission increases super linearly with the radio range, so the total energy consumption grows exponentially with node separation which results in the decrease in network lifetime. Figure 5 shows the effects of transmission range on energy consumption. From the simulation, it has been observed that the transmission range of node should be about $100 \mathrm{~m}$ in order to optimize the network lifetime. Results of E3C are better than GBS because in GBS, frequency of head rotation is quite high and all the nodes participate in cluster head re-election, thus leading to poor energy utilization and lower network life. Whereas in E3C cluster head is responsible to elect the new cluster head and only those nodes who have energy greater than threshold level, participate in cluster head re-election process. Thus, it leads to less energy consumption, hence overall network lifetime enhanced.

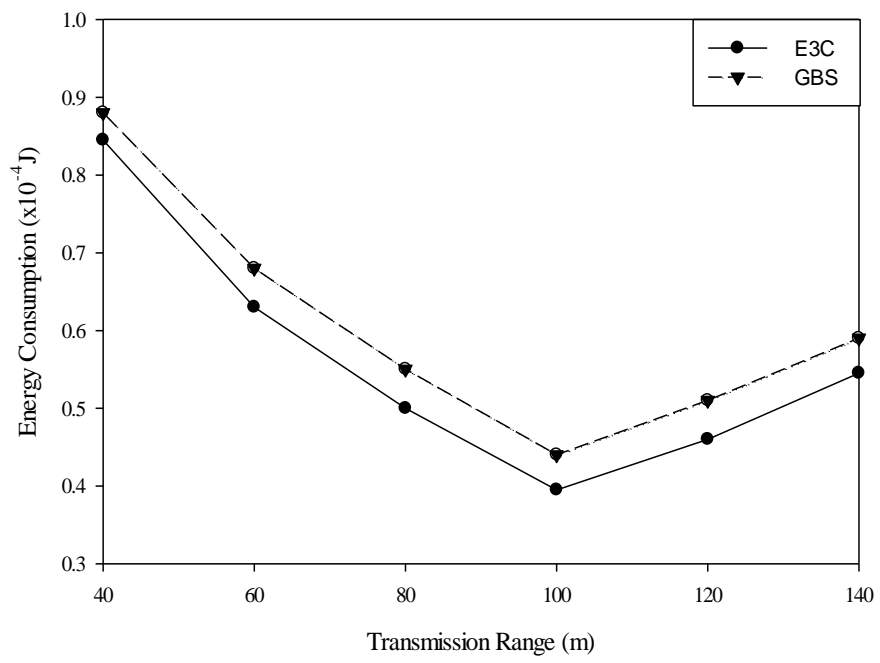

Figure 5. Effect of Transmission Range on Energy Consumption 


\section{Conclusion}

In this paper, we have proposed an energy efficient entropy based clustering (E3C) algorithm for wireless sensor network. In proposed approach, we are exploiting temporal correlation locally among the sensor nodes using entropy. we initially collect data values at grid level and process these values to find temporal correlation. Later on, existing grids are modified to form new clusters, which are more stable. Simulation results show that E3C performs well under various network scenarios

\section{References}

[1] Y. Nam, S. Rho, and B. Lee, "Intelligent context-aware energy management using the incremental simultaneous method in future wireless sensor networks and computing systems," Eurasip Journal on Wireless Communications and Networking, vol. 10, no. 1, (2013), pp. 1-11.

[2] F. Wang, S. Wu, K. Wang, and X. Hu, "Energy-Efficient Clustering Using Correlation and Random Update Based on Data Change Rate for Wireless Sensor Networks," IEEE Sensors Journal, vol. 16, no. 13, (2016), pp. 5471-5480.

[3] P. Musilek, P. Kromer, and T. Barton, "E-BACH: Entropy-based clustering hierarchy for wireless sensor networks," Proceedings - 2015 IEEE/WIC/ACM International Joint Conference on Web Intelligence and Intelligent Agent Technology, WI-IAT 2015, (2016), pp. 231-232.

[4] N. Thi, T. Nga, N. K. Khanh, and S. N. Hong, "Entropy-based Correlation Clustering for Wireless Sensor Networks in Multi-Correlated Regional Environments," vol. 5, no. 2, (2016).

[5] K. Xiao, B. Sun, C. Gui, and H. Chen, "A Novel Energy Entropy Based on Clusterhead Selection Algorithm for Wireless Sensor Networks," International Journal of Grid and Distributed Computing, vol. 9, no. 3, (2016), pp. 199-208.

[6] A. Sinha and D. K. Lobiyal, "Performance evaluation of data aggregation for cluster-based wireless sensor network," Human-centric Computing and Information Sciences, vol. 3, no. 13, (2013), pp. 1-17.

[7] T. M. Cover and J. A. Thomas, Elements of Information Theory. (2005).

[8] Y. Zhuang, J. Pan, and G. Wu, "Energy-Optimal Grid-Based Clustering in Wireless Microsensor Networks with Data Aggregation," International Journal of Parallel, Emergent and Distributed Systems, vol. 25 , no. 6, (2010), pp. 531-550. 
International Journal of Grid and Distributed Computing

Vol. 10, No. 7 (2017) 